\title{
Dysmorphology report
}

\section{Fetal brain disruption sequence: a milder variant}

\author{
Carsten G Bönnemann, Peter Meinecke
}

\begin{abstract}
We report on a female infant with severe microcephaly, scalp rugae, overlapping sutures, and telancephalic damage with relatively satisfactory early motor development. This case represents a milder form of the 'fetal brain disruption sequence', which is thought to be the result of an exogenous insult to the developing brain during the second half of gestation.
\end{abstract}

\section{History \\ Prenatal and birth. First pregnancy of a 31 year old mother. Intermittent bleeding in the fourth week of gestation: ultrasound estimation of gestational age was hampered by constantly small biparietal diameter initially. Eventually microcephaly was strongly suspected from ultrasound data. \\ Vaginal delivery in the 35th week of gestation, Apgar scores of 9, 10, and 10 at two, five, and 10 minutes, respectively. Referred to our hospital because of dysmorphic features and microcephaly.}

Family. Non-consanguineous parents. A half sib from another marriage of the father is normal.

\section{Altonaer Kinderkrankenhaus, \\ Bleickenallee 38, \\ D-2000 Hamburg 50, \\ Federal Republic of Germany. \\ C $\mathbf{G}$ Bönnemann}

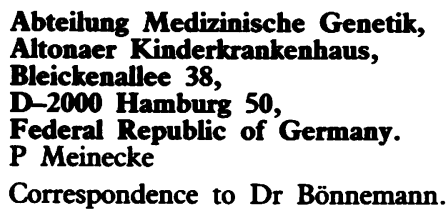

Received for publication 11 September 1989.

Revised version accepted for publication 2 November 1989.

\section{Clinical examination}

Birth weight $1670 \mathrm{~g}$ (10th centile), length $42 \mathrm{~cm}$ (10th centile). Severe microcephaly with head circumference of $24 \mathrm{~cm}$ (-6 SD), striking scalp rugae (fig 1), and overlapping coronal sutures palpable. Slight micrognathia and large appearing, well formed ears, but otherwise normal face. Apart from minor muscular hypotonia, rest of examination normal.

\section{Investigations}

Radiographs of the skull as newborn (fig 2a). Severe microcephaly, parietal bone overlapping frontal bone, other sutures abnormally narrow. No intracranial calcifications.

Computed tomography of the head as newborn (fig $2 b$ ). Grossly enlarged lateral ventricles (especially temporal horns and cellae mediae), cortical mantle thinned with rarefaction of gyral pattern. Subarachnoid space particularly marked in the frontotemporal regions. Caudal structures considered to be normal.

TORCH titres were negative.

\section{Clinical course}

General development was satisfactory, otherwise the clinical course was complicated by intermittent bradycardia, making continuous monitoring necessary. Discharge at 40 weeks' gestational age. Motor development at this age was considered to be normal by an experienced examiner. The child was seen at 1 year by the family paediatrician who was surprised by the good progress the child had made. He considered the motor development to be equivalent to a 4 month level. Unfortunately, re-examination of the child by one of us was not possible.

\section{Discussion}

The observed pattern is consistent with the 'fetal brain disruption sequence' described by Russell et al. ${ }^{1}$ Severe microcephaly, scalp rugae resembling cutis verticis gyrata, and overlapping sutures indicating 

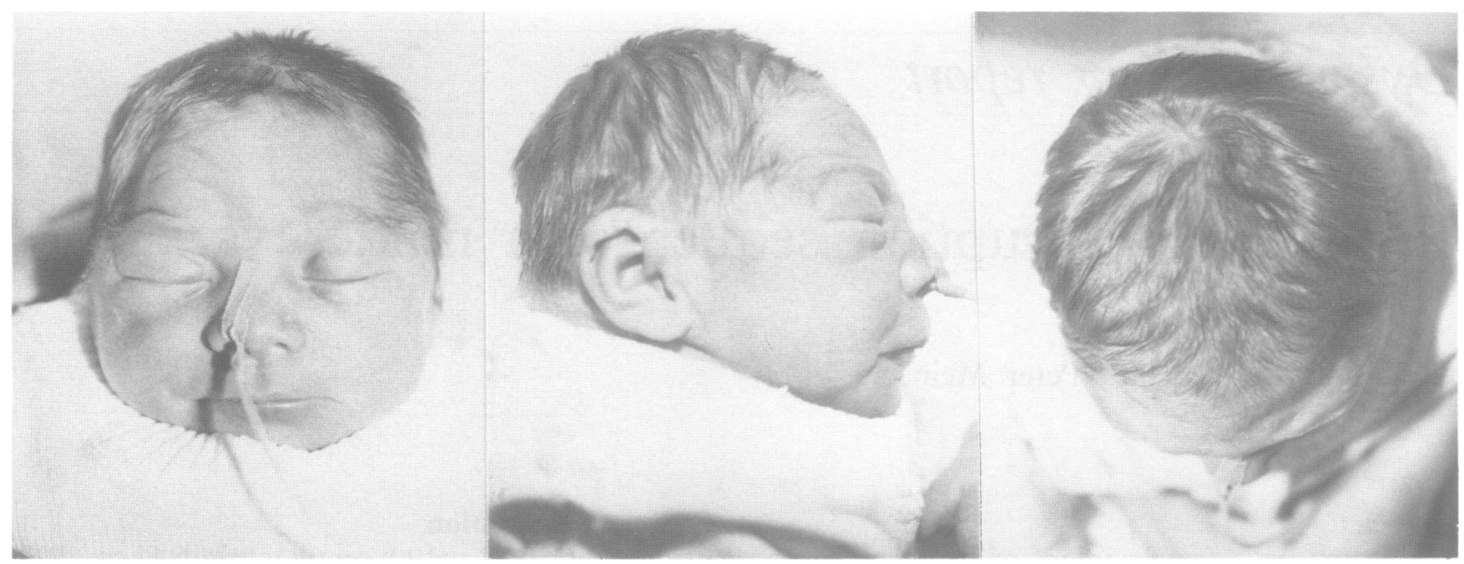

Figure 1 Patient as a newborn. Note severe microcephaly and scalp rugae.

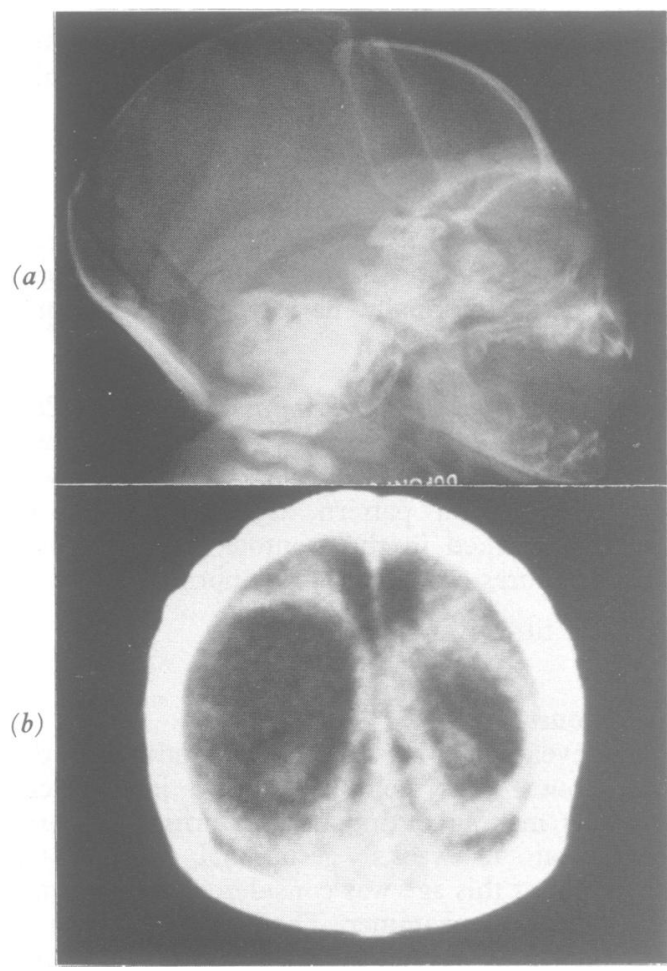

Figure 2 (a) Radiograph of the skull showing parietal bones overlapping frontal bones. (b) CT scan of the head showing grossly enlarged lateral ventricles. Note also visible rugae of the scalp.

collapse of the fetal skull owing to severe brain destruction with diminution of intracranial hydrostatic pressure are thought to be diagnostic of this type of microcephaly, distinguishing it from other, often heritable, types of severe congenital micro- cephaly. ${ }^{23}$ With regard to aetiology, a vascular insult or a prenatal infection (for example, herpes simplex ${ }^{4}$ ), disrupting normal brain development around the second to third trimester, ${ }^{1}$ are thought to be causally related to this sequence. Since TORCH titres were negative in our patient, a vascular insult could be assumed here. Our case is noteworthy in that it represents a milder variant than that described by Russell et al. ${ }^{1}$ Such variation in severity is consistent with the presumably diverse consequences of a vascular or infectious insult affecting the brain during late pregnancy.

In spite of the severe brain destruction proved by the CT scan, the early motor development in our patient appeared to be adequate. Thus, it is conceivable that the disruption, possibly of vascular aetiology, was essentially limited to the telencephalon. These structures are considered to be of minor importance in early motor development, which might explain the better prognosis for early motor development than seen in more generalised conditions, such as metabolic disorders or disturbed cytoarchitecture of the brain.

The help of Dr Ruth Engelbrecht and Dr Ulrike Pfeiffer in providing clinical data is greatly appreciated.

1 Russell LJ, Weaver DD, Bull MJ, Weinbaum M. In utero brain destruction resulting in collapse of the fetal skull, microcephaly, scalp rugae, and neurologic impairment: the fetal brain disruption sequence. Am f Med Genet 1984;17:509-21.

2 Warkany J, Lemire RJ, Cohen MM. Mental retardation and congenital malformations of the central nervous system. Chicago: Year Book Medical Publishers, 1981.

3 Tolmie JL, McNay M, Stephenson JBP, Doyle D, Connor JM. Microcephaly: genetic counselling and antenatal diagnosis after the birth of an affected child. Am f Med Genet 1987;27:583-94.

4 Baldwin S, Whitley RJ. Intrauterine herpes simplex virus infection. Teratology 1989;39:1-10. 